UFIFT-HEP-05-27

hep-ph/0510256

\title{
Viewing Lepton Mixing through the Cabibbo Haze
}

\author{
Lisa L. Everett \\ Institute for Fundamental Theory, Department of Physics \\ University of Florida, Gainesville, FL, 32611, USA
}

\begin{abstract}
We explore the hypothesis that the Cabibbo angle is an expansion parameter for lepton as well as quark mixing. Cabibbo effects are deviations from zero mixing for the quarks but are deviations from unknown mixings for the leptons, such that lepton mixing is veiled by a Cabibbo haze. We present a systematic classification of parametrizations and catalog the leading order Cabibbo effects. We find that the size of the CHOOZ angle is not always correlated with the observability of $\mathrm{CP}$ violation. This phenomenological approach has practical merit both as a method for organizing top-down flavor models and as a guideline for planning future experiments.
\end{abstract}




\section{Introduction}

The observation of neutrino oscillations provides concrete evidence that neutrinos are massive and lepton mixings are observable. Current observations do not allow for an unambiguous extraction of the neutrino mass pattern. However, the Maki-Nakagawa-Sakata-Pontecorvo (MNSP) lepton mixing matrix [1, 2] has now been measured [3, 4, 5, 6, 17, 8, 9, revealing fundamental differences between the lepton and quark mixings. The quark mixing angles of the Cabibbo-Kobayashi-Maskawa (CKM) matrix are all small, with the largest angle given by the Cabibbo angle $\theta_{c} \simeq 13^{\circ}$. In contrast, two of the lepton mixing angles are large (the atmospheric and solar angles) and one is small (the CHOOZ angle, which is not yet measured but bounded to be $\lesssim \theta_{c}[8]$ ). The challenge to understand this discrepancy provides an intriguing framework in which to explore the flavor puzzles of the SM, particularly within the context of quark-lepton grand unification [10, for which all available data can be synthesized in the search for a credible theory of flavor.

As a step toward this goal, recently a phenomenological approach was advocated in which parametrizations of the lepton mixing matrix were developed as an expansion in $\lambda \equiv \sin \theta_{c} \simeq 0.22$ [11] in analogy with Wolfenstein's parametrization of the CKM [12]. In addition to its practical advantages for phenomenology, the Wolfenstein parametrization hints at a guiding principle for flavor theory by providing a framework for examining quark mixing in the $\lambda \rightarrow 0$ limit. Extending this hypothesis to the lepton sector, one finds that the lepton mixings are unknown in the $\lambda \rightarrow 0$ limit (unlike the quark mixings, which vanish). Hence, if the limit of zero Cabibbo angle is meaningful for theory, there is a Cabibbo haze [11] in lepton mixing, in which the initial or "bare" values of the mixings are screened by Cabibbo-sized effects.

In this approach, Cabibbo effects can be deviations from zero mixing (as in the quark sector) or deviations of the mixing angles from presumably large initial values. Parametrizations of the MNSP matrix are categorized according to the bare mixings and the structure of the allowed perturbations. Perturbations which are linear in $\lambda$ yield shifts of $\lesssim \theta_{c} \simeq 13^{\circ}$ (or larger if multiple contributions shift the same parameter), while $\mathcal{O}\left(\lambda^{2}\right.$ ) shifts are $\sim 3^{\circ}$. CP violating phases can enter the $\mathcal{O}(\lambda)$ shifts but may only occur at subleading order, in which case the effective MNSP phase is suppressed and the size of the CHOOZ angle does not dictate the size of $\mathrm{CP}$-violating observables. Cabibbo haze was previously explored for the theoretically motivated class of models with a vanishing initial $\mathrm{CHOOZ}$ angle [11. The purpose of this paper is to generalize this approach to additional models and to provide a complete catalog of the dominant $\mathcal{O}(\lambda)$ effects.

Cabibbo haze allows for many Wolfenstein-like parametrizations of the MNSP matrix which are consistent with current data, although regularities should emerge upon more precise measurements. Despite these ambiguities, this classification scheme is worthwhile even at this stage of experimental observations. Ultimately, it serves as an organizing principle for categorizing the many top-down flavor models based on a $\lambda$ expansion. Using the Cabibbo angle as an expansion parameter for lepton mixing also provides a guideline for planning future experiments. Of particular interest is the program to measure the $\mathrm{CHOOZ}$ angle, for which future facilities are expected to reach the $\mathcal{O}\left(\lambda^{2}\right)$ range [13. Within this general theoretical framework, probing the CHOOZ angle at this level will provide important insight into the nature of lepton mixing in the $\lambda \rightarrow 0$ limit.

This paper is structured as follows. In Section 2, after a brief discussion of the current status of the data, we review the lessons from grand unification which form the basis of this approach. We discuss the systematics of our theoretical framework in Section 3, and turn to a discussion of the leptonic Cabibbo shifts in the mixing angles and the CP-violating phases in Section 4. Finally, we present the conclusions and outlook.

\section{Preliminaries}

We provide here a brief review of the data as well as the basic philosophy and motivation for our phenomenological approach. We begin with the lepton data. ${ }^{1}$ In the standard parametrization ([15] and references therein):

$$
\begin{aligned}
\mathcal{U}_{\mathrm{MNSP}} & =\mathcal{R}_{1}\left(\theta_{\oplus}\right) \mathcal{R}_{2}\left(\theta_{13}, \delta_{\mathrm{MNSP}}\right) \mathcal{R}_{3}\left(\theta_{\odot}\right) \mathcal{P} \\
& \equiv\left(\begin{array}{ccc}
1 & 0 & 0 \\
0 & \cos \theta_{\oplus} & \sin \theta_{\oplus} \\
0 & -\sin \theta_{\oplus} & \cos \theta_{\oplus}
\end{array}\right)\left(\begin{array}{ccc}
\cos \theta_{13} & 0 & \sin \theta_{13} e^{-i \delta_{\mathrm{MNSP}}} \\
0 & 1 & 0 \\
-\sin \theta_{13} e^{i \delta_{\mathrm{MNSP}}} & 0 & \cos \theta_{13}
\end{array}\right)\left(\begin{array}{ccc}
\cos \theta_{\odot} & \sin \theta_{\odot} & 0 \\
-\sin \theta_{\odot} & \cos \theta_{\odot} & 0 \\
0 & 0 & 1
\end{array}\right) \mathcal{P},
\end{aligned}
$$

\footnotetext{
${ }^{1}$ In this paper, we do not include constraints from the LSND reactor experiment 14, which necessitate either additional neutrino families and/or CPT violation.
} 
in which $\mathcal{R}_{i}(\theta)$ denote rotations by $\theta$ about the $i$ th axis, and $\delta_{\text {MNSP }}$ and the diagonal matrix $\mathcal{P}$ are CP-violating phases $(\mathcal{P}$ is physical only if neutrinos are Majorana fermions [16]). Combined data from solar (SNO-salt [3, 4] and Super-Kamiokande [5]), atmospheric [6], reactor [7, 8], and accelerator [9] experiments yield at $3 \sigma$ [17]:

$$
\theta_{\oplus}=45_{-10^{\circ}}^{+10^{\circ}} ; \quad \theta_{\odot}=33.9_{-2.3^{\circ}}^{\circ+2 .{ }^{\circ}} ; \quad \theta_{13}<13^{\circ} .
$$

The rather precise constraints on the solar angle are indicated by the recent SNO data 4 when combined with KamLAND results [7. The atmospheric angle is consistent with maximal mixing, while maximal solar mixing is ruled out. Presently there are no experimental constraints on the CP-violating phases. Note that the limiting value of the $\mathrm{CHOOZ}$ angle $\theta_{13}$ is approximately equal to $\theta_{c}$.

The CKM quark mixing matrix in the standard parametrization is

$$
\mathcal{U}_{\mathrm{CKM}}=\mathcal{R}_{1}\left(\theta_{23}^{\mathrm{CKM}}\right) \mathcal{R}_{2}\left(\theta_{13}^{\mathrm{CKM}}, \delta_{\mathrm{CKM}}\right) \mathcal{R}_{3}\left(\theta_{12}^{\mathrm{CKM}}\right)
$$

has three small mixing angles and one $\mathcal{O}(1) \mathrm{CP}$-violating phase [15]:

$$
\theta_{12}^{\mathrm{CKM}}=13.0^{\circ} \pm 0.1^{\circ} \simeq \theta_{c} ; \quad \theta_{23}^{\mathrm{CKM}}=2.4^{\circ} \pm 0.1^{\circ} ; \quad \theta_{13}^{\mathrm{CKM}}=0.2^{\circ} \pm 0.1^{\circ} ; \quad \delta_{\mathrm{CKM}}=60^{\circ} \pm 14^{\circ} .
$$

The CKM matrix is therefore approximately the identity matrix up to effects of order $\lambda$, as encoded by the Wolfenstein parametrization [12]:

$$
\mathcal{U}_{\mathrm{CKM}}=\left(\begin{array}{ccc}
1-\frac{\lambda^{2}}{2} & \lambda & A \lambda^{3}(\rho-i \eta) \\
-\lambda & 1-\frac{\lambda^{2}}{2} & A \lambda^{2} \\
A \lambda^{3}(1-\rho-i \eta) & -A \lambda^{2} & 1
\end{array}\right)+\mathcal{O}\left(\lambda^{4}\right),
$$

in which $\lambda$ and $A$ are well known $(\lambda=0.22, A \simeq 0.85)$, but $\rho$ and $\eta$ are less precisely determined, reflecting the uncertainty in $\delta_{\mathrm{CKM}}$ [15]. Although $\delta_{\mathrm{CKM}}$ is large (the only large angle in the quark sector), the rephasing invariant measure of $\mathrm{CP}$ violation (JDGW invariant) [18, 19, which is given by

$$
\mathcal{J}_{\mathrm{CP}}=\operatorname{Im}\left(\mathcal{U}_{\alpha i} \mathcal{U}_{\beta j} \mathcal{U}_{\beta i}^{*} \mathcal{U}_{\alpha j}^{*}\right),
$$

is suppressed by small mixing angles:

$$
J_{\mathrm{CP}}^{(\mathrm{CKM})} \simeq \sin 2 \theta_{12}^{\mathrm{CKM}} \sin 2 \theta_{23}^{\mathrm{CKM}} \sin 2 \theta_{13}^{\mathrm{CKM}} \sin \delta_{\mathrm{CKM}} \simeq A^{2} \lambda^{6} \eta \sim \mathcal{O}\left(10^{-5}\right) .
$$

From the perspective of quark-lepton unification, the discrepancy between the quark and lepton mixings provides an interesting challenge for theories of flavor. A standard approach to flavor model building (see e.g. 20] for a review) within this rather general framework is to build top-down models of the fermion mass matrices based on flavor symmetries which are typically broken at high scales, with the order parameter of the symmetry breaking set by $\lambda$. These models may or may not be restricted to a particular grand unified theory (GUT). The suppression of the neutrino masses can be linked with such high scales via the seesaw mechanism [21].

While perhaps surprising, the qualitative differences between the quark and lepton mixings are not inconsistent with grand unification. The seesaw mechanism introduces a new unitary matrix into the MNSP matrix that has no analogue in the CKM, which can provide a source for the discrepancy. To see this more clearly, recall that the mixing matrices are the product of left-handed rotations for fermions of charge $q$ as follows:

$$
\begin{aligned}
\mathcal{U}_{\mathrm{CKM}} & =\mathcal{U}_{2 / 3}^{\dagger} \mathcal{U}_{-1 / 3} \\
\mathcal{U}_{\mathrm{MNSP}} & =\mathcal{U}_{-1}^{\dagger} \widehat{\mathcal{U}}_{0} .
\end{aligned}
$$

The quark and charged lepton rotation matrices $\mathcal{U}_{q}$ are obtained from diagonalizing their associated Dirac mass matrices. The form of the neutrino rotation matrix depends on whether neutrinos are Dirac or Majorana particles. For Dirac neutrinos, $\widehat{\mathcal{U}}_{0}=\mathcal{U}_{0}$, while for the theoretically well-motivated case of the neutrino seesaw, $\widehat{\mathcal{U}}_{0}=\mathcal{U}_{0} \mathcal{F}[22,23]$. Hence, for seesaw models the MNSP matrix takes the form

$$
\mathcal{U}_{\mathrm{MNSP}}=\mathcal{U}_{-1}^{\dagger} \mathcal{U}_{0} \mathcal{F}
$$


which highlights the difference betweeen the quark and lepton mixing matrices. Models of the MNSP matrix can then be classified both by the structure of the lepton Dirac mass matrices and the number of large angles in $\mathcal{F}$ as given by the structure of the neutrino seesaw.

Within grand unification, possible connections can exist between the MNSP and CKM matrices. In one illustrative class of examples, the fermion Dirac mass matrices $\mathcal{M}^{(q)}$ obey $S U(5)$ and $S O(10)$ GUT relations based on the simplest Higgs structures and the down quark mass matrix is further assumed to be symmetric, such that $\mathcal{M}^{(-1 / 3)}=\mathcal{M}^{(-1 / 3) T} \sim \mathcal{M}^{(-1)}$ and $\mathcal{M}^{(2 / 3)} \sim \mathcal{M}^{(0)}$. In this case, the MNSP and CKM matrices are simply related [23:

$$
\mathcal{U}_{\text {MNSP }}=\mathcal{U}_{\text {CKM }}^{\dagger} \mathcal{F} \text {. }
$$

$\mathcal{F}$ must then contain two large mixing angles $\eta_{\odot}$ and $\eta_{\oplus}$. Eq. (10) implies that $\theta_{\odot} \sim \eta_{\odot} \pm \lambda \cos \eta_{\oplus}$ and $\theta_{13} \sim \lambda \sin \eta_{\oplus}$ due to the $\mathcal{O}(\lambda)$ mixing between the first and second families in $\mathcal{U}_{\mathrm{CKM}}$.

Another class of examples is motivated by the empirical fact that the measured values of the atmospheric and solar angles differ by $\sim \theta_{c}$, a relation known as quark-lepton complementarity 24, 25, 26, 27, 28, 29, 30, 31, 32, 33, 34, 35, 36, 37, 38, 39]. $\mathcal{U}_{\text {MNSP }}$ then initially can be bimaximal $\left(\eta_{\odot}=\eta_{\oplus}=45^{\circ}\right)$, with the solar angle shifted by a full-strength Cabibbo shift: $\theta_{\odot} \sim \eta_{\odot}-\theta_{c} . \theta_{13}$ can also be a Cabibbo effect (see e.g. [37).

While these examples can be motivated by flavor theories, one should keep in mind that the experimental measurements are not yet precise enough to select particular scenarios and since the values of the lepton mixing angles are cloaked from us by the Cabibbo haze (if indeed the $\lambda \rightarrow 0$ limit is meaningful for theory ${ }^{2}$ ), many possibilities exist beyond these prototype examples (see e.g. 39]). A taxonomy of parametrizations of the MNSP matrix for models in which the bare CHOOZ angle vanishes was carried out in [11. We now develop and extend this formalism to additional models based on the physics of lepton mixing in the $\lambda \rightarrow 0$ limit.

\section{Theoretical Framework}

The Wolfenstein parametrization is based on the idea that the hierarchical quark mixing angles can be understood as a $\lambda$ expansion, with

$$
\mathcal{U}_{\mathrm{CKM}}=1+\mathcal{O}(\lambda)
$$

In the lepton sector, a similar parametrization for the MNSP matrix requires a $\lambda$ expansion of the form

$$
\mathcal{U}_{\mathrm{MNSP}}=\mathcal{W}+\mathcal{O}(\lambda) \text {. }
$$

The starting matrix $\mathcal{W}$, which is dictated by the (unknown) underlying flavor theory, is then perturbed multiplicatively by a unitary matrix $\mathcal{V}(\lambda)$, which in turn is assumed to have a $\lambda$ expansion:

$$
\mathcal{V}(\lambda)=1+\mathcal{O}(\lambda)
$$

For the quarks, the starting matrix is the identity matrix and the perturbation matrix is given by Eq. (5). For the leptons, the structure of the allowed perturbations depend on the details of $\mathcal{W}$. Due to Cabibbo haze, $\mathcal{W}$ can take different forms which are characterized by the number of large angles. Neglecting Dirac CP-violating phases (they will be discussed later), the possibilities are:

- Three large angles. In this scenario, $\mathcal{W}$ is given by

$$
\begin{aligned}
\mathcal{W} & =\mathcal{R}_{1}\left(\eta_{\oplus}\right) \mathcal{R}_{2}\left(\eta_{13}\right) \mathcal{R}_{3}\left(\eta_{\odot}\right) \mathcal{P} \\
& \equiv\left(\begin{array}{ccc}
1 & 0 & 0 \\
0 & \cos \eta_{\oplus} & \sin \eta_{\oplus} \\
0 & -\sin \eta_{\oplus} & \cos \eta_{\oplus}
\end{array}\right)\left(\begin{array}{ccc}
\cos \eta_{13} & 0 & \sin \eta_{13} \\
0 & 1 & 0 \\
-\sin \eta_{13} & 0 & \cos \eta_{13}
\end{array}\right)\left(\begin{array}{ccc}
\cos \eta_{\odot} & \sin \eta_{\odot} & 0 \\
-\sin \eta_{\odot} & \cos \eta_{\odot} & 0 \\
0 & 0 & 1
\end{array}\right) \mathcal{P}
\end{aligned}
$$

in which the angles $\eta_{\oplus}, \eta_{13}$, and $\eta_{\odot}$ correspond to the bare values of the atmospheric, CHOOZ, and solar angles, respectively. $\mathcal{P}$ is a diagonal phase matrix of the form

$$
\mathcal{P}=\left(\begin{array}{ccc}
e^{i \alpha_{1}} & 0 & 0 \\
0 & e^{i \alpha_{2}} & 0 \\
0 & 0 & e^{i \alpha_{3}}
\end{array}\right),
$$

\footnotetext{
${ }^{2}$ An alternative expansion based on rational hierarchy and $S 3-S 2$ symmetry with an expansion parameter similar in spirit (though not in magnitude) to the Cabibbo angle can be found in 40.
} 
which encodes the two physical Majorana CP-violating phases $\alpha_{12} \equiv \alpha_{1}-\alpha_{2}$ and $\alpha_{23} \equiv \alpha_{2}-\alpha_{3}$. Since the CHOOZ angle is $\lesssim \theta_{c}$, the Cabibbo-sized perturbations must downshift $\eta_{13}$ into the allowed range.

- Two large angles. In this case, $\mathcal{W}$ contains two large angles $\eta_{\oplus}, \eta_{\odot}$ and a zero $\mathrm{CHOOZ}$ angle:

$$
\mathcal{W}=\mathcal{R}_{1}\left(\eta_{\oplus}\right) \mathcal{R}_{3}\left(\eta_{\odot}\right) \equiv\left(\begin{array}{ccc}
1 & 0 & 0 \\
0 & \cos \eta_{\oplus} & \sin \eta_{\oplus} \\
0 & -\sin \eta_{\oplus} & \cos \eta_{\oplus}
\end{array}\right)\left(\begin{array}{ccc}
\cos \eta_{\odot} & \sin \eta_{\odot} & 0 \\
-\sin \eta_{\odot} & \cos \eta_{\odot} & 0 \\
0 & 0 & 1
\end{array}\right) \mathcal{P}
$$

which of course follows from Eq. (14) with $\eta_{13}=0$. In this scenario, which was studied in detail in [11, both the CHOOZ angle and the JDGW invariant are generated from Cabibbo shifts.

- One large angle. $\mathcal{W}$ takes the form

$$
\mathcal{W}=\mathcal{R}_{1}\left(\eta_{\oplus}\right) \mathcal{P} \equiv\left(\begin{array}{ccc}
1 & 0 & 0 \\
0 & \cos \eta_{\oplus} & \sin \eta_{\oplus} \\
0 & -\sin \eta_{\oplus} & \cos \eta_{\oplus}
\end{array}\right) \mathcal{P}
$$

The solar and CHOOZ angles (and the JDGW invariant) arise from Cabibbo effects. Given the typical size of Cabibbo shifts, shifting the solar angle into the allowed range requires a large effect (e.g. a sum of several shifts). Note that only one Majorana phase $\alpha_{23}$ is observable in the absence of the perturbations.

- No large angles. In this case, all three mixing angles are generated by Cabibbo shifts. Given the typical size of Cabibbo effects, it is unlikely that they are the dominant source of the atmospheric angle, for which the best-fit value is consistent with maximal mixing (as we will see, even shifting the solar angle in this way requires a stretching of parameters). Therefore, we will not consider this case further in this paper.

Let us pause here to comment on Dirac phases, which in general may be present in $\mathcal{W}$. If the freedom in the SM to rephase the charged leptons is taken into account, these Dirac phases can be rotated away if one or more of the bare mixing angles is zero since the JDGW invariant identically vanishes. If $\mathcal{W}$ has three large angles, there is one physical combination of phases $\chi$ which enters in the standard parametrization through $\mathcal{R}_{2}\left(\eta_{13}\right) \rightarrow \mathcal{R}_{2}\left(\eta_{13}, \chi\right)$, in direct analogy with $\delta_{\text {MNSP }}$ in Eq. (1). However, when determining $\mathcal{U}_{\mathrm{MNSP}}$ at higher orders in $\lambda$ it is too preliminary to do this rephasing at the $\lambda=0$ level, as the bare Dirac phases can enter physical observables at higher orders in $\lambda$. For simplicity, we assume in this paper that these phases are absent, as otherwise the number of bare parameters proliferates. In doing so, we assume that Dirac CP violation is necessarily linked to Cabibbo shifts. This encompasses the main physics of models for which one or more of the bare mixing angles vanish. However, it is an extra assumption for models with three large initial mixing angles, placing constraints on the as yet unspecified underlying theory (which must then predict $\chi \rightarrow 0$ ).

A novel feature of these parametrizations of lepton mixing is that generically the perturbations do not commute with the starting matrix

$$
[\mathcal{W}, \mathcal{V}(\lambda)] \neq 0,
$$

leading to several possible implementations of the Cabibbo shifts:

- Right Cabibbo Shifts. The perturbations can be introduced as a multiplication of $\mathcal{V}(\lambda)$ on the right:

$$
\mathcal{U}_{\mathrm{MNSP}}=\mathcal{W} \mathcal{V}(\lambda)
$$

- Left Cabibbo Shifts. The perturbations can be implemented as a multiplication of $\mathcal{V}(\lambda)$ on the left:

$$
\mathcal{U}_{\mathrm{MNSP}}=\mathcal{V}(\lambda) \mathcal{W} .
$$

- Middle Cabibbo Shifts. The perturbations can be sandwiched between the rotation matrices of $\mathcal{W}$ :

$$
\mathcal{U}_{\mathrm{MNSP}}=\mathcal{R}_{1} \mathcal{V}(\lambda) \mathcal{R}_{2} \mathcal{R}_{3} \mathcal{P}
$$

or

$$
\mathcal{U}_{\mathrm{MNSP}}=\mathcal{R}_{1} \mathcal{R}_{2} \mathcal{V}(\lambda) \mathcal{R}_{3} \mathcal{P}
$$


If $\mathcal{W}$ has two large angles $\left(\eta_{13} \rightarrow 0\right)$, Eqs. (21) and (22) are equivalent. For one large angle in $\mathcal{W}\left(\eta_{13}, \eta_{\odot} \rightarrow 0\right)$, the middle and right Cabibbo shifts are redundant. $\mathcal{V}$ can also be sandwiched between $\mathcal{R}_{3}$ and $\mathcal{P}$; aside from the effects of the Majorana phases (discussed later), this case is equivalent to right shifts for $\mathcal{P}=1$.

At this stage, we pause to comment on the meaning of this classification of models into right, left, or middle Cabibbo shift scenarios. Since this classification depends on the initial parametrization of $\mathcal{W}$, it is perhaps not clear whether it encompasses all possibilities. To see that there is no loss of generality, recall that the assumption of Cabibbo haze is that the MNSP matrix has a $\lambda$ expansion

$$
\mathcal{U}_{\operatorname{MNSP}}(\lambda)=\sum_{n=0}^{\infty} \lambda^{n} W_{n}
$$

in which $W_{0} \equiv \mathcal{W}$. There is a choice in how to parametrize the Cabibbo haze; for example, it can be expressed as a right Cabibbo shift as follows:

$$
\mathcal{U}_{\mathrm{MNSP}}(\lambda)=\mathcal{W} \sum_{n=0}^{\infty} \lambda^{n}\left(\mathcal{W}^{-1} W_{n}\right) \equiv \mathcal{W} \mathcal{V}(\lambda)
$$

identifying $\mathcal{V}=\sum_{n=0}^{\infty} \lambda^{n}\left(\mathcal{W}^{-1} W_{n}\right)$. However, recalling that $\mathcal{W}$ is given by a product of rotation matrices $\mathcal{R}_{i}$ (neglecting $\mathcal{P}$ at the moment for simplicity, though it is straightforward to include it), this can also be expressed as a middle Cabibbo shift:

$$
\begin{aligned}
\mathcal{U}_{\mathrm{MNSP}}(\lambda) & =\mathcal{R}_{1} \mathcal{R}_{2} \mathcal{R}_{3} \mathcal{V}(\lambda) \\
& =\mathcal{R}_{1} \mathcal{R}_{2}\left(\mathcal{R}_{3} \mathcal{V}(\lambda) \mathcal{R}_{3}^{-1}\right) \mathcal{R}_{3} \equiv \mathcal{R}_{1} \mathcal{R}_{2} \mathcal{V}^{\prime}(\lambda) \mathcal{R}_{3}
\end{aligned}
$$

The generalization to other middle shifts and to left shifts is straightforward. Note that since $\mathcal{V}$ by assumption represents perturbations about the identity matrix

$$
\mathcal{V}(\lambda)=1+\sum_{i=1}^{\infty} \lambda^{n} V_{n}
$$

$\mathcal{V}^{\prime}$ can also be written in an analogous form:

$$
\mathcal{V}^{\prime}(\lambda)=\mathcal{R}_{3} \mathcal{V} \mathcal{R}_{3}^{-1}=1+\sum_{i=1}^{\infty} \lambda^{n}\left(\mathcal{R}_{3} V_{n} \mathcal{R}_{3}^{-1}\right) .
$$

Therefore, the decomposition into right, left, or middle shifts is meaningful for any specific choice of $\mathcal{V}$ (which may be illuminating in the context of the underlying flavor theory). For the purposes of our classification scheme, we will assume $\mathcal{V}$ takes a simple form. More precisely, we write $\mathcal{V}=e^{\mathcal{A}}$, with

$$
\mathcal{A}=\left(\begin{array}{ccc}
0 & \sum_{i=1} a_{i} \lambda^{i} & \sum_{i=1} c_{i} \lambda^{i} \\
-\sum_{i=1} a_{i}^{*} \lambda^{i} & 0 & \sum_{i=1} b_{i} \lambda^{i} \\
-\sum_{i=1} c_{i}^{*} \lambda^{i} & -\sum_{i=1} b_{i}^{*} \lambda^{i} & 0
\end{array}\right),
$$

in which $a_{i}, b_{i}$, and $c_{i}$ are $\mathcal{O}(1)$ coefficients. ${ }^{3} \mathcal{V}(\lambda)$ is written as an expansion in $\lambda$, such that e.g. through $\mathcal{O}\left(\lambda^{2}\right)$,

$$
\mathcal{V}=\left(\begin{array}{ccc}
1-\frac{\left|a_{1}\right|^{2}+\left|c_{1}\right|^{2}}{2} \lambda^{2} & a_{1} \lambda+\left(a_{2}-\frac{b_{1}^{*} c_{1}}{2}\right) \lambda^{2} & c_{1} \lambda+\left(c_{2}+\frac{a_{1} b_{1}}{2}\right) \lambda^{2} \\
-a_{1}^{*} \lambda-\left(a_{2}^{*}-\frac{b_{1} c_{1}^{*}}{2}\right) \lambda^{2} & 1-\frac{\left|a_{1}\right|^{2}+\left|b_{1}\right|^{2}}{2} \lambda^{2} & b_{1} \lambda+\left(b_{2}-\frac{a_{1}^{*} c_{1}}{2}\right) \lambda^{2} \\
-c_{1}^{*} \lambda-\left(c_{2}^{*}-\frac{a_{1}^{*} b_{1}^{*}}{2}\right) \lambda^{2} & -b_{1}^{*} \lambda-\left(b_{2}^{*}+\frac{a_{1} c_{1}^{*}}{2}\right) \lambda^{2} & 1-\frac{\left|b_{1}\right|^{2}+\left|c_{1}\right|^{2}}{2} \lambda^{2}
\end{array}\right)+\mathcal{O}\left(\lambda^{3}\right) .
$$

This expression for $\mathcal{V}$ encodes the Wolfenstein form of the CKM, which can be obtained by choosing $a_{1}=1$, $b_{2}=A, c_{3}=A\left(\rho-\frac{1}{2}-i \eta\right)$, and $b_{1}=c_{1,2}=0$, but also allows for more general perturbations. ${ }^{4}$

\footnotetext{
${ }^{3}$ We point out a change in notation here from [11, which used $\left(a, a^{\prime}, a^{\prime \prime}\right)$ to denote coefficients of $\mathcal{O}(\lambda)$ terms, $\left(b, b^{\prime}, b^{\prime \prime}\right)$ for $\mathcal{O}\left(\lambda^{2}\right)$ terms, and $\left(c, c^{\prime}, c^{\prime \prime}\right)$ for $\mathcal{O}\left(\lambda^{3}\right)$ terms.

${ }^{4}$ Note that Eq. [28] does not lead to the most general unitary matrix, because $\mathcal{A}$ can have diagonal entries which are purely imaginary, which yields additional phases in $\mathcal{V}$ than what appears in Eq. (29). These phases can be removed by global rephasings; for example, this is what is done for the CKM, which has only one observable phase. Although there is no a priori reason why such additional phase degrees of freedom should not be present in $\mathcal{V}$, for the sake of simplicity we will not include them in this paper.
} 
The shifts in the mixing angles induced by $\mathcal{V}(\lambda)$ are clearly dominated by perturbations linear in $\lambda$, which lead to shifts of at most $\sim \theta_{c} \simeq 13^{\circ}$. The $\mathcal{O}\left(\lambda^{2}\right)$ perturbations, which lead to shifts of $\sim 3^{\circ}$ or so, play a subdominant role but can be important in certain models. Therefore, it is useful to categorize models further according to the number of $\mathcal{O}(\lambda)$ perturbations [11]:

- Single shifts. These models have one $\mathcal{O}(\lambda)$ perturbation, i.e. either (i) $a_{1} \neq 0$ and $b_{1}=c_{1}=0$, (ii) $b_{1} \neq 0$ and $a_{1}=c_{1}=0$, or (iii) $c_{1} \neq 0$ and $a_{1}=b_{1}=0$.

- Double shifts. These models contain two $\mathcal{O}(\lambda)$ perturbations, again with three possibilities: (i) $a_{1}, b_{1} \neq 0$ and $c_{1}=0$, (ii) $b_{1}, c_{1} \neq 0$ and $a_{1}=0$, or (iii) $a_{1}, c_{1} \neq 0$ and $b_{1}=0$.

- Triple shifts. In these models, $a_{1}, b_{1}$, and $c_{1}$ are all nonvanishing.

Double and triple shifts can also be implemented in more complicated ways. One possibility is to assume sequential perturbations of the form $\mathcal{V}=\mathcal{V}_{1} \mathcal{V}_{2}$, in which $\mathcal{V}_{1}$ is given by Eq. (29), and $\mathcal{V}_{2}$ is given by the same expression after taking $\left\{a_{i}, b_{i}, c_{i}\right\} \rightarrow\left\{a_{i}^{\prime}, b_{i}^{\prime}, c_{i}^{\prime}\right\}$. In this case, double shift models can also be achieved when $\mathcal{V}_{1}$ and $\mathcal{V}_{2}$ are both given by single shifts (and a similar argument holds for triple shifts). Additional middle (or "mixed") Cabibbo shift scenarios can then be constructed by sandwiching $\mathcal{V}_{1}$ and $\mathcal{V}_{2}$ among the rotation matrices of $\mathcal{W}$. Such methods of incorporating double and triple shifts were mentioned in [11, but they add little to the qualitative conclusions for models in which the required shifts in the lepton mixing angles are of a natural size $\left(\sim \theta_{c}\right)$. However, given that we consider scenarios which require larger shifts, we will keep such sequential perturbations in mind in this paper because they allow for larger effects if $\mathcal{V}_{1,2}$ are both given by double or triple shifts (though of course, at leading order in $\lambda$ sequential perturbations are equivalent to a "standard" perturbation with unnaturally large coefficients).

For all models, an $\mathcal{O}(\lambda)$ entry in $\mathcal{V}(\lambda)$ gives rise to an $\mathcal{O}(\lambda)$ Cabibbo shift in the corresponding mixing angle. For example, a nonvanishing $a_{1}$ shifts the solar angle by a Cabibbo-sized effect (which may be sized by factors which depend on the bare MNSP parameters). Shifts can also be incurred from the $\mathcal{O}(\lambda)$ perturbations in other entries of $\mathcal{V}(\lambda)$; these shifts depend on the structure of $\mathcal{W}$ and the ways in which the Cabibbo shifts are introduced. For example, for the case of two large initial angles [11, single shift models with nonvanishing $b_{1}$ shift both the atmsopheric and $\mathrm{CHOOZ}$ angles for right shifts, but shift the atmospheric angle only for left and middle shifts. One of the purposes of this paper is to analyze the structure of the Cabibbo shifts for the scenarios with other choices of $\mathcal{W}$, an issue to which we now turn.

\section{Parametrizations}

In this section, we discuss the leptonic Cabibbo shifts for the Wolfenstein-like parametrizations of the MNSP matrix. We first present the shifts in the MNSP mixing angles to $\mathcal{O}(\lambda)$, assuming $\mathcal{V}(\lambda)$ is given by Eq. (29) (unless otherwise specified). The discussion of $\mathrm{CP}$ violation is deferred to the next subsection. We assume bare Dirac phases are absent, but include bare Majorana phases and phases in $\mathcal{V}(\lambda)$.

Before discussing specific models, it is important to keep in mind that there is a wide range of possible parametrizations that can be constructed by specifying the details of $\mathcal{W}$ and $\mathcal{V}(\lambda)$. While the leading order perturbations dictate the dominant Cabibbo shifts, subleading terms can also play an important role (particularly for the CP-violating effects, as discussed later). There is also a further range of possibilities for each of the given scenarios due to the experimental uncertainties in the MNSP parameters. This is particularly at play for the atmospheric and $\mathrm{CHOOZ}$ angles, which have experimental uncertainties which are roughly of $\mathcal{O}(\lambda)$. The solar angle is measured more precisely, with error bars of $\mathcal{O}\left(\lambda^{2}\right)$.

Given the wide range of scenarios consistent with current data, we choose not to analyze specific numerical examples at this stage. Particular parametrizations may emerge as compelling from the standpoint of flavor theory, warranting further analysis. Improved data will be invaluable in narrowing the range of possible parametrizations. Although improvement in the atmospheric angle is not expected in the foreseeable future [41, the planned reactor neutrino experiments, superbeams and/or neutrino factories are expected to probe the CHOOZ angle from its current $\mathcal{O}(\lambda)$ range down to the $\mathcal{O}\left(\lambda^{2}\right) \sim 3^{\circ}$ level [13]. It is therefore useful to classify scenarios according to whether they predict a CHOOZ angle of $\mathcal{O}(\lambda)$ or further suppressed. Hence, we now present a complete catalog of the $\mathcal{O}(\lambda)$ leptonic Cabibbo shifts for each scenario of interest. 


\subsection{Cabibbo-shifted lepton mixing angles}

\section{Two large angles.}

We begin by reviewing and summarizing the results for two large initial mixing angles. Given the patterns of the data as well as the philosophy of treating the Cabibbo angle as a small expansion parameter, this scenario is arguably the most plausible starting point, and has been the primary focus for most flavor model building attempts. For this reason, this class of models was first discussed as a prototype example of Cabibbo haze in [11, to which we refer the reader for more details. Here we note for reference the following general results for the $\mathcal{O}(\lambda)$ shifts in the mixing angles (including phases):

- Right shifts: $\mathcal{U}_{\mathrm{MNSP}}=\mathcal{R}_{1}\left(\eta_{\oplus}\right) \mathcal{R}_{3}\left(\eta_{\odot}\right) \mathcal{P} \mathcal{V}(\lambda)$.

$$
\begin{aligned}
\theta_{\odot} & =\eta_{\odot}+\lambda\left|a_{1}\right| \cos \left(\alpha_{12}+\phi_{a_{1}}\right)+\mathcal{O}\left(\lambda^{2}\right) \\
\theta_{\oplus} & =\eta_{\oplus}+\lambda\left(\cos \eta_{\odot}\left|b_{1}\right| \cos \left(\alpha_{23}+\phi_{b_{1}}\right)-\sin \eta_{\odot}\left|c_{1}\right| \cos \left(\alpha_{12}-\alpha_{23}+\phi_{c_{1}}\right)\right)+\mathcal{O}\left(\lambda^{2}\right) \\
\theta_{13} & =\lambda\left|b_{1} e^{i \alpha_{23}} \sin \eta_{\odot}+c_{1} e^{i\left(\alpha_{12}-\alpha_{23}\right)} \cos \eta_{\odot}\right|+\mathcal{O}\left(\lambda^{2}\right) .
\end{aligned}
$$

- Left shifts: $\mathcal{U}_{\mathrm{MNSP}}=\mathcal{V}(\lambda) \mathcal{R}_{1}\left(\eta_{\oplus}\right) \mathcal{R}_{3}\left(\eta_{\odot}\right) \mathcal{P}$.

$$
\begin{aligned}
\theta_{\odot} & =\eta_{\odot}+\lambda\left(\cos \eta_{\oplus}\left|a_{1}\right| \cos \phi_{a_{1}}-\sin \eta_{\oplus}\left|c_{1}\right| \cos \phi_{c_{1}}\right)+\mathcal{O}\left(\lambda^{2}\right) \\
\theta_{\oplus} & =\eta_{\oplus}+\lambda\left|b_{1}\right| \cos \phi_{b_{1}}+\mathcal{O}\left(\lambda^{2}\right) \\
\theta_{13} & =\lambda\left|\sin \eta_{\oplus} a_{1}+\cos \eta_{\oplus} c_{1}\right|+\mathcal{O}\left(\lambda^{2}\right) .
\end{aligned}
$$

- Middle shifts: $\mathcal{U}_{\mathrm{MNSP}}=\mathcal{R}_{1}\left(\eta_{\oplus}\right) \mathcal{V}(\lambda) \mathcal{R}_{3}\left(\eta_{\odot}\right) \mathcal{P}$.

$$
\begin{aligned}
\theta_{\odot} & =\eta_{\odot}+\lambda\left|a_{1}\right| \cos \phi_{a_{1}}+\mathcal{O}\left(\lambda^{2}\right) \\
\theta_{\oplus} & =\eta_{\oplus}+\lambda\left|b_{1}\right| \cos \phi_{b_{1}}+\mathcal{O}\left(\lambda^{2}\right) \\
\theta_{13} & =\lambda\left|c_{1}\right|+\mathcal{O}\left(\lambda^{2}\right) .
\end{aligned}
$$

These results demonstrate that the shifts in the mixing angles depend on the Majorana phases $\alpha_{12}, \alpha_{23}$ only in the right Cabibbo shift scenario. The special status of Majorana phases for right Cabibbo shifts is a general theme that will continue throughout this paper, and is a result which is easy to understand. More precisely, the presence of Majorana phases in $\mathcal{U}_{\mathrm{MNSP}}$ is due to the lack of freedom to rephase Majorana fermions, a feature which is encoded (in the standard parametrization) as a diagonal phase matrix multiplied on the far right. Since right shifts introduce the perturbations $\mathcal{V}(\lambda)$ in that position and generically

$$
[\mathcal{P}, \mathcal{V}(\lambda)] \neq 0,
$$

the right shifts can be rewritten as follows:

$$
\begin{aligned}
\mathcal{U}_{\mathrm{MNSP}} & =\mathcal{W P} \mathcal{V} \\
& =\mathcal{W}\left(\mathcal{P} \mathcal{V} \mathcal{P}^{-1}\right) \mathcal{P} \equiv \mathcal{W} \mathcal{V}_{\mathcal{M}} \mathcal{P}
\end{aligned}
$$

Eq. (40) implies that $\mathcal{V}_{\mathcal{M}}$ can be obtained from $\mathcal{V}$ through the replacements $a_{i} \rightarrow a_{i} e^{i \alpha_{12}}, b_{i} \rightarrow b_{i} e^{i \alpha_{23}}$, and $c_{i} \rightarrow c_{i} e^{i\left(\alpha_{12}-\alpha_{23}\right)}$, a result which is manifest in Eqs. (30)- (32).

Before moving on, we stress once again that for a specific form of $\mathcal{V}$, different shift scenarios can lead to very different results for the mixing angles. For example, if one assumes that $\mathcal{V}$ has the same hierarchical structure at leading order as the CKM (i.e., $b_{1}=c_{1}=0, a_{1} \neq 0$ ), models with a bimaximal starting matrix $\mathcal{W}$ (as motivated e.g. by quark-lepton complementarity) can in principle be consistent with the data for right shifts and middle shifts; note that in both cases the $\mathrm{CHOOZ}$ angle is predicted to be subleading in $\lambda$. However, the left shift scenario does not fit the data in this case, as the shift in the solar angle is sized by factors dependent on the bare angles (amounting here to a suppression). Of course, within our approach there are other ways to make this starting matrix consistent with the data, as there is no reason a priori to focus only on perturbations which resemble the CKM. For example, left shifts can also work if in addition to $\mathcal{O}(\lambda)$ mixing between the first and second generations, the perturbations have $\mathcal{O}(\lambda)$ mixing between the first and third families (see Eqs. (33)- (35)). 


\section{Three large angles: Cabibbo-downshifted CHOOZ angle.}

We now turn to the most general scenario, which has three nonvanishing angles in $\mathcal{W}$. This structure can be motivated e.g. by the idea of neutrino "anarchy" first proposed in [42, which predicts neutrino mass matrices with random $\mathcal{O}(1)$ entries, leading to three large mixing angles. Although the current bound on the $\mathrm{CHOOZ}$ angle is small, it is not vanishingly small, and hence the anarchy hypothesis can provide a viable framework for flavor model building. If neutrino anarchy can be consistently embedded within grand unification, Cabibbosized perturbations can act to reduce the CHOOZ angle from its typically large starting value, which could potentially open up new avenues for model building.

Given this form of $\mathcal{W}$ and $\mathcal{V}$ given by Eq. (29), the mixing angles for the Cabibbo shift scenarios are:

- Right shifts: $\mathcal{U}_{\mathrm{MNSP}}=\mathcal{R}_{1}\left(\eta_{\oplus}\right) \mathcal{R}_{2}\left(\eta_{13}\right) \mathcal{R}_{3}\left(\eta_{\odot}\right) \mathcal{P} \mathcal{V}(\lambda)$

$$
\begin{aligned}
\theta_{\odot}= & \eta_{\odot}+\lambda\left(\left|a_{1}\right| \cos \left(\alpha_{12}+\phi_{a_{1}}\right)-\right. \\
& \left.\tan \eta_{13}\left(\cos \eta_{\odot}\left|b_{1}\right| \cos \left(\alpha_{23}+\phi_{b_{1}}\right)+\sin \eta_{\odot}\left|c_{1}\right| \cos \left(\alpha_{12}-\alpha_{23}+\phi_{c_{1}}\right)\right)\right)+\mathcal{O}\left(\lambda^{2}\right) \\
\theta_{\oplus}= & \eta_{\oplus}+\frac{\lambda}{\cos \eta_{13}}\left(\cos \eta_{\odot}\left|b_{1}\right| \cos \left(\alpha_{23}+\phi_{b_{1}}\right)-\sin \eta_{\odot}\left|c_{1}\right| \cos \left(\alpha_{12}-\alpha_{23}+\phi_{c_{1}}\right)\right)+\mathcal{O}\left(\lambda^{2}\right) \\
\theta_{13}= & \eta_{13}+\lambda\left(\sin \eta_{\odot}\left|b_{1}\right| \cos \left(\alpha_{23}+\phi_{b_{1}}\right)+\cos \eta_{\odot}\left|c_{1}\right| \cos \left(\alpha_{12}-\alpha_{23}+\phi_{c_{1}}\right)\right)+\mathcal{O}\left(\lambda^{2}\right) .
\end{aligned}
$$

- Left shifts: $\mathcal{U}_{\mathrm{MNSP}}=\mathcal{V}(\lambda) \mathcal{R}_{1}\left(\eta_{\oplus}\right) \mathcal{R}_{2}\left(\eta_{13}\right) \mathcal{R}_{3}\left(\eta_{\odot}\right) \mathcal{P}$.

$$
\begin{aligned}
\theta_{\odot} & =\eta_{\odot}+\frac{\lambda}{\cos \eta_{13}}\left(\cos \eta_{\oplus}\left|a_{1}\right| \cos \phi_{a_{1}}-\sin \eta_{\oplus}\left|c_{1}\right| \cos \phi_{c_{1}}\right)+\mathcal{O}\left(\lambda^{2}\right) \\
\theta_{\oplus} & =\eta_{\oplus}+\lambda\left(\left|b_{1}\right| \cos \phi_{b_{1}}-\tan \eta_{13}\left(\cos \eta_{\oplus}\left|a_{1}\right| \cos \phi_{a_{1}}-\sin \eta_{\oplus}\left|c_{1}\right| \cos \phi_{c_{1}}\right)\right)+\mathcal{O}\left(\lambda^{2}\right) \\
\theta_{13} & =\eta_{13}+\lambda\left(\sin \eta_{\oplus}\left|a_{1}\right| \cos \phi_{a_{1}}+\cos \eta_{\oplus}\left|c_{1}\right| \cos \phi_{c_{1}}\right)+\mathcal{O}\left(\lambda^{2}\right) .
\end{aligned}
$$

- Middle left shifts: $\mathcal{U}_{\mathrm{MNSP}}=\mathcal{R}_{1}\left(\eta_{\oplus}\right) \mathcal{V}(\lambda) \mathcal{R}_{2}\left(\eta_{13}\right) \mathcal{R}_{3}\left(\eta_{\odot}\right) \mathcal{P}$.

$$
\begin{aligned}
\theta_{\odot} & =\eta_{\odot}+\frac{\lambda}{\cos \eta_{13}}\left|a_{1}\right| \cos \phi_{a_{1}}+\mathcal{O}\left(\lambda^{2}\right) \\
\theta_{\oplus} & =\eta_{\oplus}+\lambda\left(\left|b_{1}\right| \cos \phi_{b_{1}}-\tan \eta_{13}\left|a_{1}\right| \cos \phi_{a_{1}}\right)+\mathcal{O}\left(\lambda^{2}\right) \\
\theta_{13} & =\eta_{13}+\lambda\left|c_{1}\right| \cos \phi_{c_{1}}+\mathcal{O}\left(\lambda^{2}\right) .
\end{aligned}
$$

- Middle right shifts: $\mathcal{U}_{\mathrm{MNSP}}=\mathcal{R}_{1}\left(\eta_{\oplus}\right) \mathcal{R}_{2}\left(\eta_{13}\right) \mathcal{V}(\lambda) \mathcal{R}_{3}\left(\eta_{\odot}\right) \mathcal{P}$.

$$
\begin{aligned}
\theta_{\odot} & =\eta_{\odot}+\lambda\left(\left|a_{1}\right| \cos \phi_{a_{1}}-\tan \eta_{13}\left|b_{1}\right| \cos \phi_{b_{1}}\right)+\mathcal{O}\left(\lambda^{2}\right) \\
\theta_{\oplus} & =\eta_{\oplus}+\frac{\lambda}{\cos \eta_{13}}\left|b_{1}\right| \cos \phi_{b_{1}}+\mathcal{O}\left(\lambda^{2}\right) \\
\theta_{13} & =\eta_{13}+\lambda\left|c_{1}\right| \cos \phi_{c_{1}}+\mathcal{O}\left(\lambda^{2}\right) .
\end{aligned}
$$

As in the previous case, each Cabibbo shift scenario leads to a particular pattern of shifted mixing angles for a given $\mathcal{W}$ and $\mathcal{V}$. Since in this scenario the primary issue is to downshift the CHOOZ angle, it is instructive to compare the correlations between the dominant shifts in $\theta_{13}$ and the other angles for each scenario. Right and left shifts imply particular correlations between the shift in $\theta_{13}$ and the shifts in $\theta_{\odot}$ and $\theta_{\oplus}$ (with the right shifts involving the Majorana phases as usual). In contrast, for the two middle shift scenarios, the dominant shift in the CHOOZ angle is uncorrelated with the dominant shifts in the other two mixing angles.

There are several ways to construct viable scenarios given a particular choice of the bare angles $\eta_{\odot}, \eta_{\oplus}$, and $\eta_{13}$. Although we defer a detailed analysis of specific scenarios for future study, consider as an illustrative example a scenario in which all three bare mixing angles are close to their maximal values. In this case, both a sizeable downshift in the CHOOZ angle (of order $\gtrsim 2 \theta_{c}$ or so) and a downshift in the solar angle of $\sim \theta_{c}$ are required. Let us assume that with a suitable choice of parameters the $\mathrm{CHOOZ}$ angle can be shifted into the 
allowed range (we will return to this issue shortly). The question is then whether the solar angle can be shifted into the rather precise range allowed by the data. ${ }^{5}$

For right shifts, Eqs. (41)-(43) show that downshifting $\theta_{13}$ and $\theta_{\odot}$ either implies particular shifts (e.g. in the atmospheric angle) or dictates the sizes of other coefficients (see Eq. (41D). In addition, since this scenario has a tendency toward anticorrelation between the shifts of the solar and CHOOZ angles, in general the parameters must be stretched for the ratio of these two shifts to be within the ballpark of the data, independently of whether the large shift in the CHOOZ angle can be achieved without fine-tuning. For left shifts, an inspection of Eqs. (44)-(46) shows that the required ratio of the downshifts of the solar and CHOOZ angles is not as difficult to achieve. For both middle shift scenarios the size of the dominant shift in the CHOOZ angle is unconnected with the shift in the solar angle, so independent coefficients govern the ratio of their shifts.

In this example, the bare $\mathrm{CHOOZ}$ angle is very different from its experimental value, requiring a large Cabibbo shift for consistency with the data. Such large shifts can be difficult to achieve for perturbations of the form given in Eq. (29) with $\mathcal{O}(1)$ coefficients. Obtaining large shifts can be facilitated by assuming sequential perturbations (as mentioned previously). Sequential perturbations also allow for many additional mixed Cabibbo shift scenarios, corresponding to the many ways to introduce these two perturbation matrices in the MNSP matrix. We do not enumerate all the possibilities here, but rather comment that such scenarios may be of theoretical interest because they can more easily make a "trimaximal" starting matrix consistent with the data. We will return to this idea when discussing models in which the solar angle is sourced by Cabibbo effects.

Of course, trimaximal or other starting matrices which require a large shift in the CHOOZ angle are not the only option. For perturbations of the usual type (Eq. (29) without unnaturally large coefficients), it is much easier to accommodate scenarios in which the initial CHOOZ angle is perhaps only $\sim \theta_{c}$ away from the experimental bound. For example, Cabibbo haze also allows for the possibility of models in which the initial solar and $\mathrm{CHOOZ}$ angles are similar, which may be an interesting avenue for model building.

We also point out out that the CHOOZ angle is expected to be close to the experimental bound within this class of models, since Cabibbo effects have a characteristic size. (Note that this is true for neutrino anarchy models in general, though without additional effects such as Cabibbo haze such models are somewhat disfavored even with the current bound.) Future experiments, which will either measure $\theta_{13}$ or push the bound to the $\mathcal{O}\left(\lambda^{2}\right)$ region, will be crucial in determining whether such models remain viable options for flavor model building.

\section{One large angle: Cabibbo shifted solar angle.}

In this class of models, $\mathcal{W}$ is assumed to contain only the large initial atmospheric angle $\eta_{\oplus}$. This scenario has attractive features from the perspective of flavor model building, as it is relatively easy to obtain a structure of this type in the context of three family mixing (for example, it occurs for mass matrices with a degenerate row or column). In contrast, it is considerably more difficult to generate a pattern of two large angles and one small angle without fine-tuning in three family models (see e.g. [22] for discussions of this point).

However, the price to pay in this scenario is that the solar angle must experience a large shift in order to be consistent with the data. Given that this large shift is larger than the characteristic size of the perturbations, this scenario requires that the solar angle receives several Cabibbo shifts which then sum to a large effect. Assuming $\mathcal{V}(\lambda)$ of the form given by Eq. (29), the shifts are given by

- Right shifts: $\mathcal{U}_{\mathrm{MNSP}}=\mathcal{R}_{1}\left(\eta_{\oplus}\right) \mathcal{P} \mathcal{V}(\lambda)$.

$$
\begin{aligned}
\theta_{\odot} & =\lambda\left|a_{1}\right|+\mathcal{O}\left(\lambda^{2}\right) \\
\theta_{\oplus} & =\eta_{\oplus}+\lambda\left|b_{1}\right| \cos \left(\alpha_{23}+\phi_{b_{1}}\right)+\mathcal{O}\left(\lambda^{2}\right) \\
\theta_{13} & =\lambda\left|c_{1}\right|+\mathcal{O}\left(\lambda^{2}\right) .
\end{aligned}
$$

- Left shifts: $\mathcal{U}_{\mathrm{MNSP}}=\mathcal{V}(\lambda) \mathcal{R}_{1}\left(\eta_{\oplus}\right) \mathcal{P}$.

$$
\begin{aligned}
\theta_{\odot} & =\lambda\left|a_{1} \cos \eta_{\oplus}-c_{1} \sin \eta_{\oplus}\right|+\mathcal{O}\left(\lambda^{2}\right) \\
\theta_{\oplus} & =\eta_{\oplus}+\lambda\left|b_{1}\right| \cos \phi_{b_{1}}+\mathcal{O}\left(\lambda^{2}\right) \\
\theta_{13} & =\lambda\left|a_{1} \sin \eta_{\oplus}+c_{1} \cos \eta_{\oplus}\right|+\mathcal{O}\left(\lambda^{2}\right) .
\end{aligned}
$$

${ }^{5}$ Note that $\mathcal{O}\left(\lambda^{2}\right)$ terms should also be included since large shifts are required, although they are not displayed here. 
Eqs. (53) and (56) show that it is difficult to obtain a large enough shift in solar angle with $\mathcal{O}(1)$ coefficients. This scenario thus requires either large coefficients, which is contrary to the philosophy of treating $\lambda$ as an expansion parameter, or more complicated scenarios such as sequential perturbations $\mathcal{V} \sim \mathcal{V}_{1} \mathcal{V}_{2}$ (in which both $\mathcal{V}_{1}$ and $\mathcal{V}_{2}$ are at least double shift models). The latter approach leads to the following additional possibilities:

- Right shifts: $\mathcal{U}_{\mathrm{MNSP}}=\mathcal{R}_{1}\left(\eta_{\oplus}\right) \mathcal{P} \mathcal{V}_{1}(\lambda) \mathcal{V}_{2}(\lambda)$

$$
\begin{aligned}
\theta_{\odot} & =\lambda\left|a_{1}+a_{1}^{\prime}\right|+\mathcal{O}\left(\lambda^{2}\right) \\
\theta_{\oplus} & =\eta_{\oplus}+\lambda\left(\left|b_{1}\right| \cos \left(\alpha_{23}+\phi_{b_{1}}\right)+\left|b_{1}^{\prime}\right| \cos \left(\alpha_{23}+\phi_{b_{1}^{\prime}}\right)\right)+\mathcal{O}\left(\lambda^{2}\right) \\
\theta_{13} & =\lambda\left|c_{1}+c_{1}^{\prime}\right|+\mathcal{O}\left(\lambda^{2}\right) .
\end{aligned}
$$

- Left shifts: $\mathcal{U}_{\mathrm{MNSP}}=\mathcal{V}_{1}(\lambda) \mathcal{V}_{2}(\lambda) \mathcal{R}_{1}\left(\eta_{\oplus}\right) \mathcal{P}$.

$$
\begin{aligned}
\theta_{\odot} & =\lambda\left|\left(a_{1}+a_{1}^{\prime}\right) \cos \eta_{\oplus}-\left(c_{1}+c_{1}^{\prime}\right) \sin \eta_{\oplus}\right|+\mathcal{O}\left(\lambda^{2}\right) \\
\theta_{\oplus} & =\eta_{\oplus}+\lambda\left(\left|b_{1}\right| \cos \phi_{b_{1}}+\left|b_{1}^{\prime}\right| \cos \phi_{b_{1}^{\prime}}\right)+\mathcal{O}\left(\lambda^{2}\right) \\
\theta_{13} & =\lambda\left|\left(c_{1}+c_{1}^{\prime}\right) \cos \eta_{\oplus}+\left(a_{1}+a_{1}^{\prime}\right) \sin \eta_{\oplus}\right|+\mathcal{O}\left(\lambda^{2}\right)
\end{aligned}
$$

- Middle shifts: $\mathcal{U}_{\mathrm{MNSP}}=\mathcal{V}_{1}(\lambda) \mathcal{R}_{1}\left(\eta_{\oplus}\right) \mathcal{P} \mathcal{V}_{2}(\lambda)$

$$
\begin{aligned}
\theta_{\odot} & =\lambda\left|e^{i \alpha_{12}} a_{1}^{\prime}+a_{1} \cos \eta_{\oplus}-c_{1} \sin \eta_{\oplus}\right|+\mathcal{O}\left(\lambda^{2}\right) \\
\theta_{\oplus} & =\eta_{\oplus}+\lambda\left(\left|b_{1}\right| \cos \phi_{b_{1}}+\left|b_{1}^{\prime}\right| \cos \left(\alpha_{23}+\phi_{b_{1}^{\prime}}\right)\right)+\mathcal{O}\left(\lambda^{2}\right) \\
\theta_{13} & =\lambda\left|e^{i\left(\alpha_{12}-\alpha_{23}\right)} c_{1}^{\prime}+c_{1} \cos \eta_{\oplus}+a_{1} \sin \eta_{\oplus}\right|+\mathcal{O}\left(\lambda^{2}\right) .
\end{aligned}
$$

These middle shifts are hybrid scenarios (a left shift of $\mathcal{V}_{1}$ and a right shift of $\mathcal{V}_{2}$ ), which are different than the middle shifts discussed previously for models with two or more large initial angles and $\mathcal{V}=\mathcal{V}_{1}$.

In this class of models the primary issue is to achieve the necessary shift in the solar angle to boost it into the experimentally measured range. Hence, it is of interest to examine the correlations between this large shift and the shifts in the other mixing angles. The prediction for the CHOOZ angle is of particular interest, since it must receive a smaller Cabibbo shift than the solar angle. For right shifts, Eqs. (59)-(61) demonstrate that the shifts in the mixing angles are uncorrelated at leading order. The shifts of the solar and CHOOZ angles are correlated for both left and middle shifts (though the atmospheric angle is not), but with a tendency toward anticorrelation so these remain viable scenarios.

From the perspective of the data, this class of models is arguably the least plausible Cabibbo haze scenario of the several we have considered. In general, shifting the solar angle to its relatively large experimental value through effects of Cabibbo size places nontrivial constraints on the nature and details of the allowed perturbations, although it may still be a worthwhile avenue of exploration for flavor model building.

\subsection{CP violation}

In a three-family mixing scheme, it is well known that if neutrinos are Majorana fermions, CP violation in the lepton mixing matrix can occur not only from a Dirac phase $\delta_{\mathrm{MNSP}}$, which is in direct analogy with the quark mixing phase $\delta_{\mathrm{CKM}}$, but also from two Majorana phases. The Majorana phases do not contribute to the JDGW invariant defined in Eq. (6), but their effects can be encoded through two additional rephasing invariants chosen from the following set [3]:

$$
\mathcal{S}_{\mathrm{CP} \alpha i j}=\operatorname{Im}\left(\mathcal{U}_{\alpha i} \mathcal{U}_{\alpha j}^{*} \xi_{j}^{*} \xi_{i}\right),
$$

in which the $\xi_{i}$ are given by a generalized Majorana condition

$$
C \bar{\nu}_{j}^{T}=\xi_{j}^{* 2} \nu_{j} .
$$

Majorana phases only contribute to lepton number violating processes such as neutrinoless double beta decay and neutrino-antineutrino oscillations (see e.g. 44, 45 for discussions). These processes are helicity suppressed and difficult to observe. From the standpoint of our phenomenological approach, the dependence on the neutrino masses $m_{i}$ (via the helicity suppression factors) adds a new facet to the analysis, in that $m_{i}$ also may be governed 
by a $\lambda$ expansion. Note that as our analysis has been independent of the masses so far, we have not specified any details about their properties in the $\lambda \rightarrow 0$ limit. For these reasons, we defer such an analysis to a future study and focus instead in this paper on Dirac CP violation.

For Dirac CP violation, the questions to be addressed in our phenomenological approach include how the phase $\delta_{\mathrm{MNSP}}$ is generated in $\mathcal{U}_{\mathrm{MNSP}}$ and what are the resulting predictions for the lepton sector JDGW invariant

$$
\mathcal{J}_{\mathrm{CP}} \simeq \sin 2 \theta_{\oplus} \sin 2 \theta_{\odot} \sin 2 \theta_{13} \sin \delta_{\mathrm{MNSP}}
$$

The answers hinge upon the nature of $\mathrm{CP}$ violation in the $\lambda \rightarrow 0$ limit. As previously mentioned, the starting matrix $\mathcal{W}$ may have a number of Dirac phases. These phases are not physical in the $\lambda \rightarrow 0$ limit if any of the bare mixing angles are zero (see Eq. (6) ). If $\mathcal{W}$ has three large angles, one phase combination $\chi$ is physical in the absence of the perturbations, in which case $\delta_{\mathrm{MNSP}} \sim \chi$ at leading order. However, even the phases which are unobservable in the $\lambda \rightarrow 0$ limit may provide a source of CP violation once Cabibbo effects are switched on and all three mixing angles are nonvanishing.

For simplicity, and given that our phenomenological approach does not address the origin of the physics of the $\lambda \rightarrow 0$ limit, we will not elaborate further on scenarios in which $\mathcal{W}$ contains bare Dirac phases. We focus instead on cases where these phases are absent, but allow for bare Majorana phases and phases in the Cabibbo-sized perturbations. In this case, there are two possible ways to generate $\delta_{\text {MNSP: }}$

- Generating $\delta_{\mathrm{MNSP}}$ from complex $\mathcal{V}(\lambda)$. In this case, $\mathcal{V}(\lambda)$ is the source of CP-violating phases. We assume that these phases can naturally be $\mathcal{O}(1)$ (as in the CKM). Models in this class can be categorized in terms of whether CP violation enters at leading or higher order in $\lambda$, and whether the effective MNSP phase is predicted to be $\mathcal{O}(1)$ or further suppressed.

- Generating $\delta_{\mathrm{MNSP}}$ from Majorana phases. Majorana phases can also provide a source for Dirac CP violation once the Cabibbo-sized perturbations are switched on in particular scenarios. For left and middle Cabibbo shifts, $\mathcal{P}$ does not contribute to the JDGW invariant (by inspection). However, Majorana phases do contribute to the JDGW invariant for right Cabibbo shifts, as such shifts encode $\mathcal{P}$ through the modification $\mathcal{V} \rightarrow \mathcal{V}_{\mathcal{M}}$ as shown in Eq. (40).

\section{Example: Revisiting $\mathcal{U}_{\mathrm{MNSP}}=\mathcal{U}_{\mathrm{CKM}}^{\dagger} \mathcal{F}$.}

To illustrate these points, we now present a representative example of theoretical interest, in which $\mathcal{W}$ has two large angles and the hierarchical structure of $\mathcal{V}(\lambda)$ is similar to that of the CKM matrix. ${ }^{6}$ Specifically, we focus here on the model with $\mathcal{U}_{\mathrm{MNSP}}=\mathcal{U}_{\mathrm{CKM}}^{\dagger} \mathcal{F}$ (see Eq. (10) and surrounding discussion). This model was also discussed in [11, but we will do so in slightly more general terms here. First recall that in this model, which is a left shift scenario with $\mathcal{V}=\mathcal{U}_{\mathrm{CKM}}^{\dagger}$ and $\mathcal{F}=\mathcal{W}=\mathcal{R}_{1}\left(\eta_{\oplus}\right) \mathcal{R}_{3}\left(\eta_{\odot}\right) \mathcal{P}$, the shifts in the angles are given by

$$
\begin{aligned}
\theta_{\odot} & =\eta_{\odot}-\lambda \cos \eta_{\oplus}+\mathcal{O}\left(\lambda^{3}\right) \\
\theta_{\oplus} & =\eta_{\oplus}-\lambda^{2}\left(A+\frac{1}{4} \sin 2 \eta_{\oplus}\right)+\mathcal{O}\left(\lambda^{3}\right) \\
\theta_{13} & =-\lambda \sin \eta_{\oplus}+\mathcal{O}\left(\lambda^{3}\right) .
\end{aligned}
$$

As this model is a left shift scenario, the dominant shift in $\theta_{13}$ is $\sim \lambda$ due to the $\mathcal{O}(\lambda)$ mixing between the first and second families in the quark sector (in addition, the Majorana phases do not enter the Cabibbo shifts).

Using the Wolfenstein form of $\mathcal{U}_{\mathrm{CKM}}$ as given in Eq. (5), the JDGW invariant for $\mathcal{U}_{\mathrm{MNSP}}$ is (see also [11]):

$$
\mathcal{J}_{\mathrm{CP}}=\frac{1}{4} A \lambda^{3} \eta \cos \eta_{\oplus} \sin 2 \eta_{\oplus} \sin 2 \eta_{\odot} .
$$

Given that this model has one $\mathcal{O}(\lambda)$ and two $\mathcal{O}(1)$ mixing angles, Eq. (74) indicates that $\delta_{\text {MNSP }} \sim \mathcal{O}\left(\lambda^{2}\right)$, in contrast to the $\mathcal{O}(1)$ CKM phase $\delta_{\mathrm{CKM}}$. This suppression occurs because the phases in $\mathcal{V}$ are only manifest in subdominant contributions to the mixing angles. As discussed in [11, models with this feature demonstrate that

\footnotetext{
${ }^{6}$ See also 39 for examples in which the MNSP phase enters directly in the Cabibbo haze correction to the solar angle.
} 
while the size of $\theta_{13}$ is clearly correlated with the prospects for the observability of lepton sector CP violation, it does not tell the whole story because the CP-violating phase itself may be additionally suppressed.

Eq. (74) was obtained using a very specific form of $\mathcal{U}_{\mathrm{CKM}}$, in which the freedom to rotate away phases which are unphysical in the Standard Model has already been taken into account and the remaining phase degree of freedom was chosen to appear at a particular location in the CKM matrix. For the quarks, this does not lead to any loss of generality as the JDGW invariant is independent of this choice by construction. However, it is too preliminary to restrict the CKM to this form when computing the JDGW invariant for the leptons within this class of models, and indeed the location of the CP-violating phase(s) affects the results. To see this more clearly, let us write the CKM in the form given in Eqs. (28)-(29), leaving the coefficients $a_{1}, b_{2}$, and $c_{3}$ general rather than specifying the Wolfenstein values. While the JDGW invariant for $\mathcal{U}_{\text {CKM }}^{\dagger}$ is

$$
\mathcal{J}_{\mathrm{CP}}^{\left(\mathrm{CKM}^{\dagger}\right)}=-\lambda^{6} \operatorname{Im}\left(a_{1} b_{2} c_{3}^{*}\right)
$$

which shows the convention independence explicitly, the JDGW invariant for $\mathcal{U}_{\mathrm{MNSP}}=\mathcal{U}_{\mathrm{CKM}}^{\dagger} \mathcal{F}$ is

$$
\begin{aligned}
\mathcal{J}_{\mathrm{CP}} & =\lambda \operatorname{Im}\left(a_{1}\right) \sin \eta_{\oplus} \sin 2 \eta_{\oplus} \sin 2 \eta_{\odot}\left(1-\frac{\lambda^{2}}{4}\left|a_{1}\right|^{2}\right)+\frac{\lambda^{3}}{4} \operatorname{Im}\left(c_{3}\right) \cos \eta_{\oplus} \sin 2 \eta_{\oplus} \sin 2 \eta_{\odot} \\
& +\frac{\lambda^{3}}{8} \operatorname{Im}\left(a_{1} b_{2}\right) \sin \eta_{\oplus}\left(1-3 \cos 2 \eta_{\oplus}\right) \sin 2 \eta_{\odot}-\frac{\lambda^{3}}{4} \operatorname{Im}\left(a_{1} b_{2}^{*}\right) \cos \eta_{\oplus} \sin 2 \eta_{\oplus} \sin 2 \eta_{\odot}
\end{aligned}
$$

The lepton JDGW invariant is $\mathcal{O}\left(\lambda^{3}\right)$ if $\operatorname{Im}\left(a_{1}\right)=0$ and either $\operatorname{Im}\left(b_{2}\right) \neq 0$ or $\operatorname{Im}\left(c_{3}\right) \neq 0$ (note that Eq. (76) reduces to Eq. (74) for the Wolfenstein parameters $\left.\operatorname{Im}\left(a_{1}\right)=0, \operatorname{Im}\left(b_{2}\right)=0, \operatorname{Im}\left(c_{3}\right)=-A \eta\right)$. However, if $\operatorname{Im}\left(a_{1}\right) \neq 0$, the JDGW invariant is $\mathcal{O}(\lambda)$ and the effective $\delta_{\text {MNSP }}$ is $\mathcal{O}(1)$, because in this case a CP-violating phase enters in the dominant shift of the CHOOZ angle. Hence, whether the JDGW invariant is $\mathcal{O}(\lambda)$ or $\mathcal{O}\left(\lambda^{3}\right)$ in this model will depend on the details of the associated model for $\mathcal{U}_{\mathrm{CKM}}$.

The lesson to be learned from this exercise is a point which was also stressed in 11]: the location of the phases in $\mathcal{V}$, and whether they enter in the dominant or subdominant shifts in the mixing angles, plays an important

role in determining the size of Dirac CP violation. With this in mind, we now turn to the a discussion of the JDGW invariants for the different Cabibbo haze scenarios. For economy of presentation, we present the leading order in $\lambda$ results for each scenario of interest and do not display higher order contributions. Within our range of assumptions, this corresponds to scenarios in which $\delta_{\text {MNSP }}$ is $\mathcal{O}(1)$ in most cases. The exception is the class of models with three large initial mixing angles, in which case the leading order effects correspond to $\delta_{\text {MNSP }} \sim \mathcal{O}(\lambda)$ since we have explicitly chosen not to consider situations in which there are bare Dirac phases.

\section{Two large angles.}

Once again, we begin with the Cabibbo haze scenario in which $\mathcal{W}$ has two large angles, which was also studied in the absence of bare Majorana phases in 11] (to which we refer the reader for details).

- Right shifts: $\mathcal{U}_{\mathrm{MNSP}}=\mathcal{R}_{1}\left(\eta_{\oplus}\right) \mathcal{R}_{3}\left(\eta_{\odot}\right) \mathcal{P} \mathcal{V}(\lambda)$.

$$
\mathcal{J}_{\mathrm{CP}}=-\frac{1}{4} \lambda \sin 2 \eta_{\oplus} \sin 2 \eta_{\odot}\left(\sin \eta_{\odot}\left|b_{1}\right| \sin \left(\alpha_{23}+\phi_{b_{1}}\right)+\cos \eta_{\odot}\left|c_{1}\right| \sin \left(\alpha_{12}-\alpha_{23}+\phi_{c_{1}}\right)\right)+\mathcal{O}\left(\lambda^{2}\right) .
$$

- Left shifts: $\mathcal{U}_{\mathrm{MNSP}}=\mathcal{V}(\lambda) \mathcal{R}_{1}\left(\eta_{\oplus}\right) \mathcal{R}_{3}\left(\eta_{\odot}\right) \mathcal{P}$.

$$
\mathcal{J}_{\mathrm{CP}}=-\frac{1}{4} \lambda \sin 2 \eta_{\oplus} \sin 2 \eta_{\odot}\left(\sin \eta_{\oplus}\left|a_{1}\right| \sin \phi_{a_{1}}+\cos \eta_{\oplus}\left|c_{1}\right| \sin \phi_{c_{1}}\right)+\mathcal{O}\left(\lambda^{2}\right) .
$$

- Middle shifts: $\mathcal{U}_{\mathrm{MNSP}}=\mathcal{R}_{1}\left(\eta_{\oplus}\right) \mathcal{V}(\lambda) \mathcal{R}_{3}\left(\eta_{\odot}\right) \mathcal{P}$.

$$
\mathcal{J}_{\mathrm{CP}}=-\frac{1}{4} \lambda \sin 2 \eta_{\oplus} \sin 2 \eta_{\odot}\left|c_{1}\right| \sin \phi_{c_{1}}+\mathcal{O}\left(\lambda^{2}\right) .
$$

The leading order contributions to the JDGW invariant are $\mathcal{O}(\lambda)$, which is to be expected since the CHOOZ angle is by construction a Cabibbo effect in this class of models. Note that as expected, the right shifts depend on the Majorana phases, allowing for an $\mathcal{O}(\lambda) \mathrm{CP}$ violation even if the leading order perturbations do not 
explicitly involve CP-violating phases. In all cases, the effective $\delta_{\mathrm{MNSP}}$ is $\mathcal{O}(1)$ unless these leading order contributions vanish for any given scenario, in which case $\delta_{\text {MNSP }}$ is further suppressed. We refer the reader to 11] for examples of each of these scenarios and additional discussion.

\section{Three large angles.}

We now turn to the case of three large angles, keeping in mind that since we do not consider the possibility of bare Dirac CP-violating phases, the leading order contributions to the JDGW invariant are of $\mathcal{O}(\lambda)$. They are given for each scenario as follows:

- Right shifts: $\mathcal{U}_{\mathrm{MNSP}}=\mathcal{R}_{1}\left(\eta_{\oplus}\right) \mathcal{R}_{2}\left(\eta_{13}\right) \mathcal{R}_{3}\left(\eta_{\odot}\right) \mathcal{P} \mathcal{V}(\lambda)$.

$$
\begin{aligned}
\mathcal{J}_{\mathrm{CP}}= & \frac{1}{2} \lambda\left[\left|a_{1}\right| \sin 2 \eta_{\oplus} \sin \eta_{13} \cos ^{2} \eta_{13} \sin \left(\alpha_{12}+\phi_{a_{1}}\right)+\right. \\
& \left|b_{1}\right| \sin \left(\alpha_{23}+\phi_{b_{1}}\right) \cos \eta_{13} \cos \eta_{\odot}\left(\sin 2 \eta_{\oplus}\left(\cos ^{2} \eta_{\odot} \sin ^{2} \eta_{13}-\sin \eta_{\odot}^{2}\right)+\sin \eta_{13} \sin 2 \eta_{\odot} \cos 2 \eta_{\oplus}\right)+ \\
& \left.\left|c_{1}\right| \sin \left(\alpha_{12}-\alpha_{23}+\phi_{c_{1}}\right) \cos \eta_{13} \sin \eta_{\odot}\left(\sin 2 \eta_{\oplus}\left(\sin ^{2} \eta_{\odot} \sin ^{2} \eta_{13}-\cos \eta_{\odot}^{2}\right)-\sin \eta_{13} \sin 2 \eta_{\odot} \cos 2 \eta_{\oplus}\right)\right] \\
& +\mathcal{O}\left(\lambda^{2}\right) .
\end{aligned}
$$

- Left shifts: $\mathcal{U}_{\mathrm{MNSP}}=\mathcal{V}(\lambda) \mathcal{R}_{1}\left(\eta_{\oplus}\right) \mathcal{R}_{2}\left(\eta_{13}\right) \mathcal{R}_{3}\left(\eta_{\odot}\right) \mathcal{P}$.

$$
\begin{aligned}
\mathcal{J}_{\mathrm{CP}}= & -\frac{1}{2} \lambda \cos \eta_{13}\left[\left|a_{1}\right| \sin \phi_{a_{1}} \cos \eta_{13} \cos \eta_{\oplus} \sin 2 \eta_{\odot}-\frac{1}{2}\left|b_{1}\right| \sin \phi_{b_{1}} \sin 2 \eta_{13} \sin 2 \eta_{\odot}\right. \\
& \left.-\left(\left|a_{1}\right| \sin \phi_{a_{1}} \cos \eta_{\oplus}-\left|c_{1}\right| \sin \phi_{c_{1}} \sin \eta_{\oplus}\right)\left(\cos 2 \eta_{\odot} \sin 2 \eta_{\oplus} \sin \eta_{13}+\sin 2 \eta_{\odot}\left(\cos \eta_{\oplus}^{2}-\sin \eta_{13}^{2} \sin \eta_{\oplus}^{2}\right)\right)\right] \\
& +\mathcal{O}\left(\lambda^{2}\right) .
\end{aligned}
$$

- Middle left shifts: $\mathcal{U}_{\mathrm{MNSP}}=\mathcal{R}_{1}\left(\eta_{\oplus}\right) \mathcal{V}(\lambda) \mathcal{R}_{2}\left(\eta_{13}\right) \mathcal{R}_{3}\left(\eta_{\odot}\right) \mathcal{P}$.

$$
\begin{aligned}
\mathcal{J}_{\mathrm{CP}}= & \frac{1}{2} \lambda \cos \eta_{13}\left[\left|a_{1}\right| \sin \phi_{a_{1}} \sin \eta_{13}\left(\sin 2 \eta_{\odot} \cos 2 \eta_{\oplus} \sin \eta_{13}+\cos 2 \eta_{\odot} \sin 2 \eta_{\oplus}\right)\right. \\
& \left.+\frac{1}{2}\left(\left|b_{1}\right| \sin \phi_{b_{1}} \sin 2 \eta_{\odot} \sin 2 \eta_{13} \cos 2 \eta_{\oplus}-\left|c_{1}\right| \sin \phi_{c_{1}} \cos \eta_{13}^{2} \sin 2 \eta_{\odot} \cos 2 \eta_{\oplus}\right)\right]+\mathcal{O}\left(\lambda^{2}\right) .
\end{aligned}
$$

- Middle right shifts: $\mathcal{U}_{\mathrm{MNSP}}=\mathcal{R}_{1}\left(\eta_{\oplus}\right) \mathcal{R}_{2}\left(\eta_{13}\right) \mathcal{V}(\lambda) \mathcal{R}_{3}\left(\eta_{\odot}\right) \mathcal{P}$.

$$
\begin{aligned}
\mathcal{J}_{\mathrm{CP}}= & \frac{1}{2} \lambda \cos \eta_{13}\left[\frac{1}{2}\left|a_{1}\right| \sin \phi_{a_{1}} \sin 2 \eta_{13} \sin 2 \eta_{\oplus} \cos 2 \eta_{\odot}+\left|b_{1}\right| \sin \phi_{b_{1}} \sin \eta_{13}\left(\cos 2 \eta_{\odot} \sin 2 \eta_{\oplus} \sin \eta_{13}\right.\right. \\
& \left.\left.+\sin 2 \eta_{\odot}\left(\cos \eta_{\oplus}^{2}-\sin \eta_{13}^{2} \sin \eta_{\oplus}^{2}\right)\right)-\cos \eta_{13}^{2} \sin 2 \eta_{\odot} \sin \eta_{\oplus}\left(\left|b_{1}\right| \sin \phi_{b_{1}} \sin \eta_{13} \sin \eta_{\oplus}+\left|c_{1}\right| \sin \phi_{c_{1}} \cos \eta_{\oplus}\right)\right] \\
& +\mathcal{O}\left(\lambda^{2}\right) .
\end{aligned}
$$

Note that Eqs. (80)- (83) reduce to their appropriate counterparts of Eqs. (777)-(79) in the limit that $\eta_{13} \rightarrow 0$.

\section{One large angle.}

Finally, we present the leading order JDGW invariants for the class of models in which the bare solar and CHOOZ angles are zero. These contributions are of $\mathcal{O}\left(\lambda^{2}\right)$, which is expected since two of the three MNSP mixing angles are due to Cabibbo effects, and therefore correspond to an effective $\delta_{\text {MNSP }}$ of $\mathcal{O}(1)$. For perturbations given by Eq. (29), the JDGW invariants are:

- Right shifts: $\mathcal{U}_{\mathrm{MNSP}}=\mathcal{R}_{1}\left(\eta_{\oplus}\right) \mathcal{P} \mathcal{V}(\lambda)$.

$$
\mathcal{J}_{\mathrm{CP}}=-\frac{1}{2} \lambda^{2} \sin 2 \eta_{\oplus}\left|a_{1} c_{1}\right| \sin \left(\alpha_{23}-\phi_{a_{1}}+\phi_{c_{1}}\right)+\mathcal{O}\left(\lambda^{3}\right) .
$$

- Left shifts: $\mathcal{U}_{\mathrm{MNSP}}=\mathcal{V}(\lambda) \mathcal{R}_{1}\left(\eta_{\oplus}\right) \mathcal{P}$. 


$$
\mathcal{J}_{\mathrm{CP}}=\frac{1}{2} \lambda^{2}\left|a_{1} c_{1}\right| \sin \left(\phi_{a_{1}}-\phi_{c_{1}}\right)+\mathcal{O}\left(\lambda^{3}\right) .
$$

We also present the JDGW invariants for perturbations of the form $\mathcal{V}=\mathcal{V}_{1} \mathcal{V}_{2}$ :

- Right shifts: $\mathcal{U}_{\mathrm{MNSP}}=\mathcal{R}_{1}\left(\eta_{\oplus}\right) \mathcal{P} \mathcal{V}_{1}(\lambda) \mathcal{V}_{2}(\lambda)$

$$
\begin{aligned}
\mathcal{J}_{\mathrm{CP}}= & -\frac{1}{2} \lambda^{2} \sin 2 \eta_{\oplus}\left[\left|a_{1} c_{1}\right| \sin \left(\alpha_{23}-\phi_{a_{1}}+\phi_{c_{1}}\right)+\left|a_{1}^{\prime} c_{1}\right| \sin \left(\alpha_{23}-\phi_{a_{1}^{\prime}}+\phi_{c_{1}}\right)\right. \\
& \left.+\left|a_{1} c_{1}^{\prime}\right| \sin \left(\alpha_{23}-\phi_{a_{1}}+\phi_{c_{1}^{\prime}}\right)+\left|a_{1}^{\prime} c_{1}^{\prime}\right| \sin \left(\alpha_{23}-\phi_{a_{1}^{\prime}}+\phi_{c_{1}^{\prime}}\right)\right]+\mathcal{O}\left(\lambda^{3}\right) .
\end{aligned}
$$

- Left shifts: $\mathcal{U}_{\mathrm{MNSP}}=\mathcal{R}_{1}\left(\eta_{\oplus}\right) \mathcal{P} \mathcal{V}_{1}(\lambda) \mathcal{V}_{2}(\lambda)$

$$
\begin{aligned}
\mathcal{J}_{\mathrm{CP}}= & \frac{1}{2} \lambda^{2}\left[\left|a_{1} c_{1}\right| \sin \left(\phi_{a_{1}}-\phi_{c_{1}}\right)-\left|a_{1}^{\prime} c_{1}\right| \sin \left(\phi_{c_{1}}-\phi_{a_{1}^{\prime}}\right)+\right. \\
& \left.\left|a_{1} c_{1}^{\prime}\right| \sin \left(\phi_{a_{1}}-\phi_{c_{1}^{\prime}}\right)+\left|a_{1}^{\prime} c_{1}^{\prime}\right| \sin \left(\phi_{a_{1}^{\prime}}-\phi_{c_{1}^{\prime}}\right)\right]+\mathcal{O}\left(\lambda^{3}\right) .
\end{aligned}
$$

- Middle shifts: $\mathcal{U}_{\mathrm{MNSP}}=\mathcal{V}_{1}(\lambda) \mathcal{R}_{1}\left(\eta_{\oplus}\right) \mathcal{P} \mathcal{V}_{2}(\lambda)$

$$
\begin{aligned}
\mathcal{J}_{\mathrm{CP}}= & \frac{1}{2} \lambda^{2} \sin 2 \eta_{\oplus}\left[\sin \eta_{\oplus}\left(\left|a_{1} a_{1}^{\prime}\right| \sin \left(\alpha_{12}-\phi_{a_{1}}+\phi_{a_{1}^{\prime}}\right)+\left|c_{1} c_{1}^{\prime}\right| \sin \left(\alpha_{12}-\alpha_{23}-\phi_{c_{1}}+\phi_{c_{1}^{\prime}}\right)\right)+\right. \\
& \cos \eta_{\oplus}\left(\left|c_{1} a_{1}^{\prime}\right| \sin \left(\alpha_{12}-\phi_{c_{1}}+\phi_{a_{1}^{\prime}}\right)-\left|a_{1} c_{1}^{\prime}\right| \sin \left(\alpha_{12}-\alpha_{23}-\phi_{a_{1}}+\phi_{c_{1}^{\prime}}\right)\right)+ \\
& \left.\left|a_{1} c_{1}\right| \sin \left(\phi_{a_{1}}-\phi_{c_{1}}\right)-\left|a_{1}^{\prime} c_{1}^{\prime}\right| \sin \left(\phi_{a_{1}^{\prime}}-\phi_{c_{1}^{\prime}}\right)\right]+\mathcal{O}\left(\lambda^{3}\right) .
\end{aligned}
$$

Note that for such sequential perturbations, the JDGW invariants for the middle as well as right shifts depends on the bare Majorana phases. This is easily understood because these middle shift scenarios are a hybrid of right shifts (which involve the Majorana phases) and left shifts (which don't).

We conclude by stressing a point mentioned in [11] which is worth repeating here. Our catalog focuses on the leading order contributions to the JDGW invariants, which usually lead to an $\mathcal{O}(1) \delta_{\mathrm{MNSP}}$ (similar to $\delta_{\mathrm{CKM}}$ ). However, since leading order contributions can be absent, it is not automatic that the MNSP phase is a large angle. Many models lead to a suppressed $\delta_{\text {MNSP }}$; for example, this occurs for left and middle shifts (and right shifts if bare Majorana phases vanish) if the dominant shifts are manifestly real. In such cases, a nonvanishing $\mathrm{CHOOZ}$ angle is a necessary but not sufficient condition for the prospects for observing leptonic CP violation.

\section{Conclusions}

The recent experimental progress in the lepton sector has revealed new avenues for exploration in the search to formulate a compelling theory of the masses and mixings of the SM fermions. As a step toward this elusive goal, we have advocated a phenomenological approach which proposes that both lepton and quark mixings can be understood as expansions in the Cabibbo angle $\lambda$. This hypothesis can be argued in the context of grand unification, and more generally in theoretical frameworks in which the flavor structures of the quarks and leptons are controlled by the same order parameter. Within this approach, the lepton mixings are enveloped in a haze of Cabibbo-sized effects, as (unlike the quark mixings) they are unknown in the $\lambda \rightarrow 0$ limit.

To aid in viewing lepton mixing through the Cabibbo haze, this paper provides a systematic classification of possible parametrizations which includes a catalog of the $\mathcal{O}(\lambda)$ effects on the mixing angles and the CP-violating phases. Although present experimental constraints are not sufficient to single out a particular parametrization, this phenomenological approach has practical applications both in categorizing top-down flavor models and in suggesting a roadmap for future measurements of the MNSP matrix. Should the limit of zero Cabibbo angle prove to be meaningful for theory, with improved data we may be able to discern the underlying flavor theory through the Cabibbo haze. 


\section{Acknowledgments}

We thank D. Chung, L. Duffy, K. Matchev, P. Ramond, and C. Thorn for helpful discussions. We also thank S. Petcov for the suggestion to include Majorana phases, as well as many other helpful comments. This work is supported by the U. S. Department of Energy under grant DE-FG02-97ER41209, and and a L'Oréal for Women in Science Postdoctoral Fellowship.

\section{References}

[1] Z. Maki, M. Nakagawa and S. Sakata, Prog. Theor. Phys. 28, 870 (1962).

[2] B. Pontecorvo, Sov. Phys. JETP 6, 429 (1957) [Zh. Eksp. Teor. Fiz. 33, 549 (1957)].

[3] The SNO Collaboration, S.N. Ahmed et al., Phys.Rev.Lett. 92 (2004) 181301.

[4] The SNO Collaboration, B. Aharmim et al. nucl-ex/0502021.

[5] The Super-Kamiokande Collaborations, S. Fukuda et al., Phys.Lett.B 539 (2002) 179.

[6] The Super-Kamiokande Collaborations, S. Fukuda et al., Phys.Rev.Lett. 81 (1998) 1562; ibid 85 (2000) 3999.

[7] The KamLAND Collaboration, K. Eguchi et al., Phys.Rev.Lett. 90 (2003) 021802.

[8] The CHOOZ Collaboration, M. Apollonio et al., Phys.Lett. B 338 (1998) 383; ibid 420 (1998) 397; ibid 466 (1999) 415; the Palo Verde collaboration, F. Boehm et al., Phys. Rev. Lett. 84, 3764 (2000) hep-ex/9912050. For improved treatments leading to significant relaxation of the upper bound see M. Apollonio et al., Eur.Phys.J. C27 (2003) 331.

[9] The K2K Collaboration, M.H. Ahn et al., Phys.Rev.Lett. 90 (2003) 041801.

[10] J. C. Pati and A. Salam, Phys. Rev. D 10, 275 (1974).

[11] A. Datta, L. Everett and P. Ramond, Phys. Lett. B 620, 42 (2005) hep-ph/0503222.

[12] L. Wolfenstein, Phys. Rev. Lett. 51, 1945 (1983).

[13] M. Freund, P. Huber and M. Lindner, Nucl. Phys. B 615, 331 (2001) hep-ph/0105071; P. Huber, M. Lindner and W. Winter, Nucl.Phys. B 645 (2002) 3; M. Apollonio et al., hep-ph/0210192 P. Huber et al., Nucl.Phys. B 665 (2003) 487; S. Pakvasa and J.W.F. Valle, Proc. Indian Natl. Sci. Acad. 70A, 189 (2004) hep-ph/0301061; V. Barger, D. Marfatia and K. Whisnant, hep-ph/0308123 and references therein; "White Paper Report on Using Nuclear Reactors to Search for a Value of $\theta_{13}$ ", K. Anderson et al., January, 2004, document available at http://www.hep.anl.gov/minos/reactor13/white.html

[14] C. Athanassopoulos et al. [LSND Collaboration], Phys. Rev. Lett. 81, 1774 (1998) nucl-ex/9709006.

[15] S. Eidelman et al., Phys. Lett. B 592, 1 (2004).

[16] S. M. Bilenky, J. Hosek and S. T. Petcov, Phys. Lett. B 94, 495 (1980).

[17] M. Maltoni et al., New J.Phys. 6 (2004) 122; M. C. Gonzalez-Garcia and M. Maltoni, hep-ph/0406056 S. Goswami, A. Bandyopadhyay and S. Choubey, hep-ph/0409224.

[18] C. Jarlskog, Phys. Rev. Lett. 55, 1039 (1985).

[19] I. Dunietz, O. W. Greenberg and D. d. Wu, Phys. Rev. Lett. 55, 2935 (1985).

[20] G. Altarelli and F. Feruglio, New J. Phys. 6, 106 (2004) hep-ph/0405048. 
[21] M. Gell-Mann, P. Ramond, and R. Slansky in Sanibel Talk, CALT-68-709, Feb 1979 retropreprinted as hep-ph/9809459, and in Supergravity (North Holland, Amsterdam 1979); T. Yanagida, in Proceedings of the Workshop on Unified Theory and Baryon Number of the Universe, KEK, Japan, Feb 1979. P. Minkowski, Phys. Lett. B 67, 421 (1977).

[22] A. Datta, F. S. Ling and P. Ramond, Nucl. Phys. B 671, 383 (2003) hep-ph/0306002.

[23] P. Ramond, hep-ph/0401001 hep-ph/0405176 Nucl. Phys. Proc. Suppl. 137, 317 (2004) hep-ph/0411009; hep-ph/0411010

[24] H. Minakata and A. Y. Smirnov, Phys. Rev. D 70, 073009 (2004) hep-ph/0405088.

[25] A. Y. Smirnov, hep-ph/0402264

[26] M. Raidal, Phys. Rev. Lett. 93, 161801 (2004) hep-ph/0404046.

[27] S. T. Petcov and W. Rodejohann, Phys. Rev. D 71, 073002 (2005) hep-ph/0409135.

[28] J. Ferrandis and S. Pakvasa, Phys. Lett. B 603, 184 (2004) hep-ph/0409204.

[29] J. Ferrandis and S. Pakvasa, Phys. Rev. D 71, 033004 (2005) hep-ph/0412038.

[30] T. Ohlsson and G. Seidl, Nucl. Phys. B 643, 247 (2002) hep-ph/0206087.

[31] C. Giunti and M. Tanimoto, Phys. Rev. D 66, 053013 (2002) hep-ph/0207096.

[32] W. Rodejohann, Phys. Rev. D 69, 033005 (2004) hep-ph/0309249.

[33] P. H. Frampton, S. T. Petcov and W. Rodejohann, Nucl. Phys. B 687, 31 (2004) hep-ph/0401206.

[34] P. H. Frampton and R. N. Mohapatra, JHEP 0501, 025 (2005) hep-ph/0407139.

[35] K. Cheung, S. K. Kang, C. S. Kim and J. Lee, hep-ph/0503122.

[36] S. K. Kang, C. S. Kim and J. Lee, Phys. Lett. B 619, 129 (2005) hep-ph/0501029.

[37] S. Antusch, S. F. King and R. N. Mohapatra, Phys. Lett. B 618, 150 (2005) hep-ph/0504007.

[38] T. Ohlsson, Phys. Lett. B 622, 159 (2005) hep-ph/0506094.

[39] S. Antusch and S. F. King, hep-ph/0508044

[40] P. Kaus and S. Meshkov, Phys. Lett. B 611, 147 (2005) hep-ph/0410024.

[41] T. Kajita, talk given at NOON-2004, Tokyo, Feb. 2004.

[42] L. J. Hall, H. Murayama and N. Weiner, Phys. Rev. Lett. 84, 2572 (2000) hep-ph/9911341.

[43] J. F. Nieves and P. B. Pal, Phys. Rev. D 36, 315 (1987).

[44] S. M. Bilenky, S. Pascoli and S. T. Petcov, Phys. Rev. D 64, 053010 (2001) hep-ph/0102265.

[45] A. de Gouvea, B. Kayser and R. N. Mohapatra, Phys. Rev. D 67, 053004 (2003) hep-ph/0211394. 